\title{
Evénements liés au passage du méristème de l'état végétatif à l'état reproducteur, chez le colza, Brassica napus. L. var. oleifera Metzg
}

\author{
M.H. Netzer ${ }^{1}$, E.D. Tittonnel ${ }^{2}$ et A. Merrien ${ }^{1}$ \\ 1 CNRS, Département Etudes et Recherches, CETIOM, 174, av. Victor-Hugo 75116 Paris; \\ 2 ENITA, 21, bd Olivier-de-Serres, B.P. 48, 21800 Quetigny, France
}

(reçu le 30-3-1988, accepté le 29-11-1988)

Résumé - Cette étude de la croissance et du développement inflorescentiel de Brassica napus L. var. oleifera, en conditions contrôlées du phytotron de Gif-sur-Yvette, a mis en évidence la présence de différents phénomènes liés à la floraison.

Nous avons constaté une modification de la morphologie foliaire, une diminution des plastochrones réels et apparents, de la longueur finale des feuilles ainsi que de leur vitesse d'allongement. L'ensemble de ces phénomènes apparaît comme partie intégrante d'un processus plus complexe mais parfaitement synchronisé. Nos résultats mettent en évidence la présence d'une feuille (appellée feuille $\alpha$ ) caractéristique du passage à la floraison et à laquelle nous nous sommes particulièrement intéressés.

Les données peuvent constituer un premier pas vers la mise au point d'un modèle permettant de prédire le nombre final de feuilles basé sur le numéro d'ordre de la feuille $\alpha$ et tenant compte de l'effet des conditions culturales du colza.

Brassica napus - floraison - feuille $\alpha$

Summary - Events connected to the change from a growth development to an inflorescentiel one on Brassica napus L. var. oleifera Metzg. This study on the Brassica napus $L$. var. oleifera Metzg growth and inflorescentiel development, under the controlled conditions of Gif-sur-Yvette phytotron, enabled the demonstration of the presence of differents phenomena related to flowering.

We have observed a modification of the leaf morphology, a decrease of the real and apparent plastochron, of the final length of leaves as well as their rate of lengthening. A special leaf (called $\alpha$ ), typical of the flowering, has been noted. This leaf can have agronomic impact. It has permitted as to establish a mathematic model useful to predict the final leaf number of the mean axis as the potential ramification number. These data constitute a first step towards the establishment of a predictive model of the yield.

Brassica napus - flowering - $\alpha$ leaf

\section{Introduction}

Les travaux réalisés sur les transformations cytologiques et morphologiques qui se déroulent, au sein du méristème, lors du passage de l'état végétatif à l'état reproducteur par Bernier et al. (1981) et Netzer et al. (1987) tendent à privilégier un instant critique de cette évolution : l'initiation florale. Les principaux acquis de ces travaux montrent que des températures inférieures ou égales à $17^{\circ} \mathrm{C}$ permettent l'initiation; de plus, plus la température est basse, plus la durée nécessaire pour la vernalisation est courte. Le nombre maximum de feuilles est obtenu pour une photopériode de $16 \mathrm{~h}$. Ici, l'accent sera mis essentielle- ment sur l'aspect dynamique des phénomènes qui interviennent au cours du développement végétatif et floral de $B$. napus et les modifications qui surviennent en recherchant des corrélations et des synchronisations afin de mieux comprendre le fonctionnement global de la plante.

\section{Matériel et Méthodes}

\section{La plante et les cultivars}

Trois cultivars différents de $B$. napus $L$. var. oleifera, largement cultivés dans les régions tempérées pour leur intérêt agronomique et pour la qualité de leur pro- 
duit, ont été choisis en raison de leurs besoins variables en vernalisation.

Ces cultivars sont :

1) Crésor, un cultivar de printemps sans besoins en vernalisation; 2) Jet neuf, cultivar d'hiver à faibles besoins; 3) Darmor, autre cultivar d'hiver mais à besoins plus élevés.

\section{Conditions générales de culture}

Les résultats présentés ici sont issus d'un travail réalisé au Phytotron de Gif-sur-Yvette et ayant pour but d'explorer les possibilités de floraison des trois cultivars (Netzer, 1986).

Après une germination sur papier filtre pendant $24 \mathrm{~h}$, les jeunes plantules sont séparées en deux lots : 1) lot à vernalisation «préalable»: elle se passe à l'obscurité en boîtes de Pétri à $1^{\circ} \mathrm{C}$. Les durées sont de : a) 1 et 2 semaines pour Crésor ; b) 2, 4, 6 et 8 semaines pour Jet Neuf ; c) 2, 6, 8, 10 et 12 semaines pour Darmor.

Au sortir de la chambre froide, les plantes les plus développées sont au stade cotylédonnaire. Le reste du cycle se déroule en chambres de culture dans les conditions suivantes:

- Crésor et Darmor : $22^{\circ} \mathrm{C}$ soit en jours continus uniquement;

- Jet Neuf : $22^{\circ} \mathrm{C}$ soit en jours continus soit en $16 \mathrm{~h}$ de jour.

2) lot à vernalisation «parallèle": ces plantes passent directement en chambre de culture où sont combinées des températures de 5,17 ou $22^{\circ} \mathrm{C}$ avec des conditions de jour continu ou de durée de jour de $9 \mathrm{~h}$.

Les températures de 5 et $17^{\circ} \mathrm{C}$ permettent l'acquisition de la vernalisation pendant la croissance (Netzer, 1986).

L'ensemble représente 33 expériences.

\section{Observations}

Au cours de cette étude, différentes observations portant sur l'appareil végétatif et sur le méristème apical ont été réalisées.

Lâge foliaire. II tient compte du nombre total de feuilles pleinement étalées $(n)$. La feuille qui émerge est affectée d'une décimale correspondant à son degré de déploiement.

Dans notre cas, l'émergence d'une feuille a été divisée en 5 étapes : 1) $n$, la feuille $n$ est pleinement étalée; 2) $n+0,25$, la feuille $n+1$ est visible; 3) $n+0,50$, la feuille $n+1$ est repliée sur elle-même; 4) $n+0,75$, la. feuille $n+1$ commence à se déplier; 5) $n+1$, la feuille $n+1$ est pleinement étalée.

Cette échelle de notation a été utilisée sur blé par Malvoisin (1984). Elle permet ainsi de connaître la fréquence d'émergence des feuilles (phyllochrone).

Le nombre total de feuilles. II englobe tout ce qui a été, est, ou sera une feuille, depuis les cicatrices jusqu'aux primordia visibles à la loupe.

Ce comptage répété au cours du temps permet de connaître le plastochrone réel.

L'état d'évolution de l'apex. Nous avons repris les stades décrits par Tittonel et al. (1982) en portant une attention particulière à deux étapes importantes : 1) le début du gonflement de l'apex en dôme (stade 2); 2) la formation des couples feuilles/méristèmes ou c.f.m (stade 3). Ce stade est pris comme définition de l'initiation florale.

\section{Les plantes observées}

Pour chacune des 33 expériences des plantes ont été disséquées ou simplement suivies.

Plantes suivies. Dès le stade cotylédonnaire, 5 plants de colza pris au hasard sont identifiés. Pendant la phase végétative, tous les 2 ou 3 jours, on mesure la longueur de chacune de leurs feuilles et on note leur âge foliaire. A l'anthèse, on enregistre le nombre final de feuilles sur l'axe principal, la forme de chacune et le niveau d'apparition des ramifications florifères.

Plantes disséquées. A la même fréquence jusqu'à la formation des fleurs nous relevons l'âge foliaire, le nombre total de feuilles et le stade de l'apex.

\section{Résultats expérimentaux}

\section{Morphologie foliaire}

\section{Longueur finale et forme}

La longueur et la forme sont en relation avec l'âge de la plante.

Les plantes à développement végétatif (Photo 1.) ne possèdent qu'un seul type de feuilles caractérisé par une complexité croissante et dont le limbe est bordé de dents arrondies. Le pétiole s'ornemente peu à peu de nombreux folioles. Cette feuille est de type lyrée pennatisequée.

Quelle que soit la durée de l'expérience (parfois plusieurs mois) toutes les feuilles sont semblables.

La taille est liée à la forme. Plus le numéro d'ordre de la feuille augmente, plus celle-ci est complexe (photo 1) et plus elle est grande (Fig. 1).

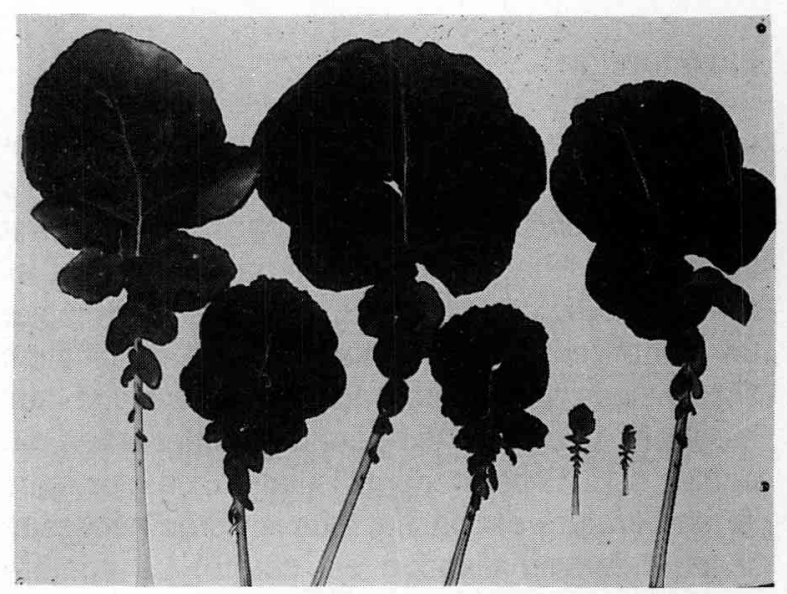

Photo 1. Morphologie foliaire des plantes à développement strictement végétatif. 
feuille $\alpha$
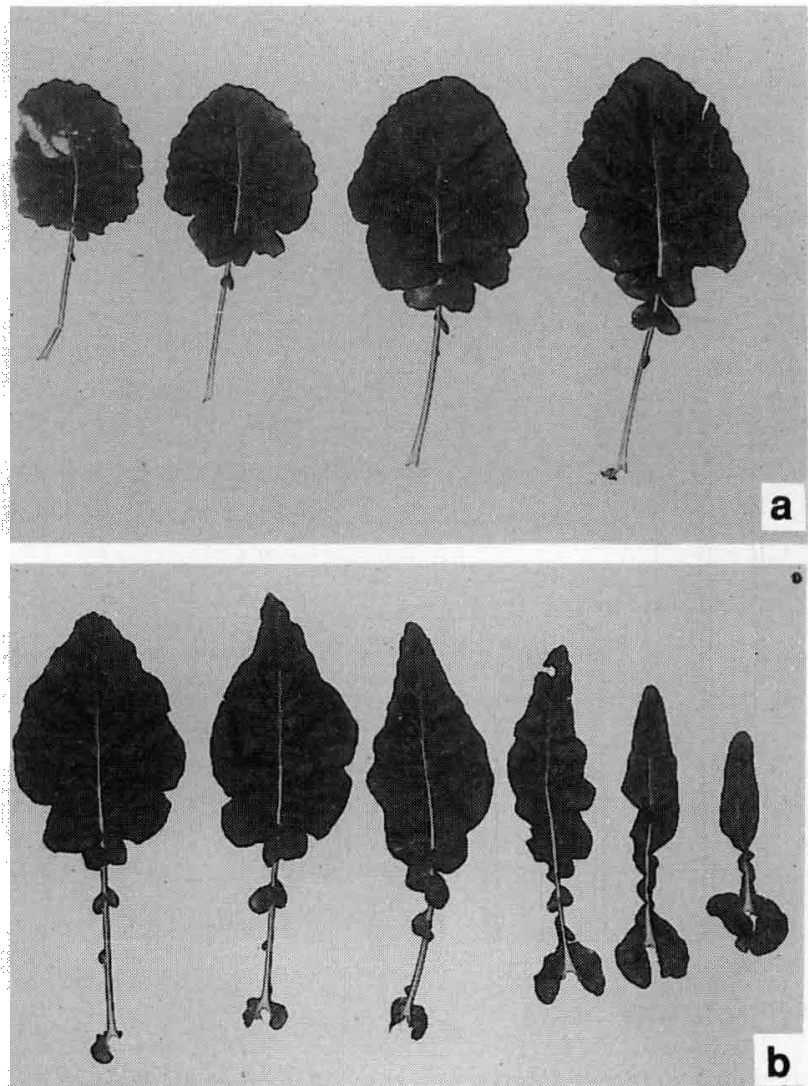

Photo 2. Morphologie foliaire des plantes à développement inflorescentiel. a. Feuilles lyrées. b. Feuilles lancéolées.
En revanche, les plantes devenant inflorescentielles présentent deux types de feuilles (Photo 2.). Les premières sont semblables à celle des plantes végétatives puis apparaît une feuille dont l'extrémité du limbe se rétrécit insensiblement en pointe. Cette feuille particulière sera appelée feuille $\alpha$. Elle marque le début de la deuxième série.

A la base du pétiole, des stipules de plus en plus engainants se développent. Sur le pétiole, les petits folioles grandissent, la feuille devient sessile et les dents disparaissent. La feuille est désormais lancéolée; il s'agit là d'un cas classique d'hétéroblastie.

La taille est liée à la forme : la forme se simplifie et la longueur diminue. La feuille $\alpha$, à la charnière des deux types, est la plus longue (Fig. 2).

\section{Vitesse d'allongement des feuilles}

Nous retrouvons les mêmes distinctions.

Chez les plantes à développement strictement végétatif, la vitesse d'allongement, calculée dans la partie linéaire de la courbe de croissance sigmoïde, augmente puis se stabilise à une valeur maximale moyenne (Fig. 3A).

En revanche pour les plantes à développement inflorescentiel (Fig. 3B), la vitesse d'allon-

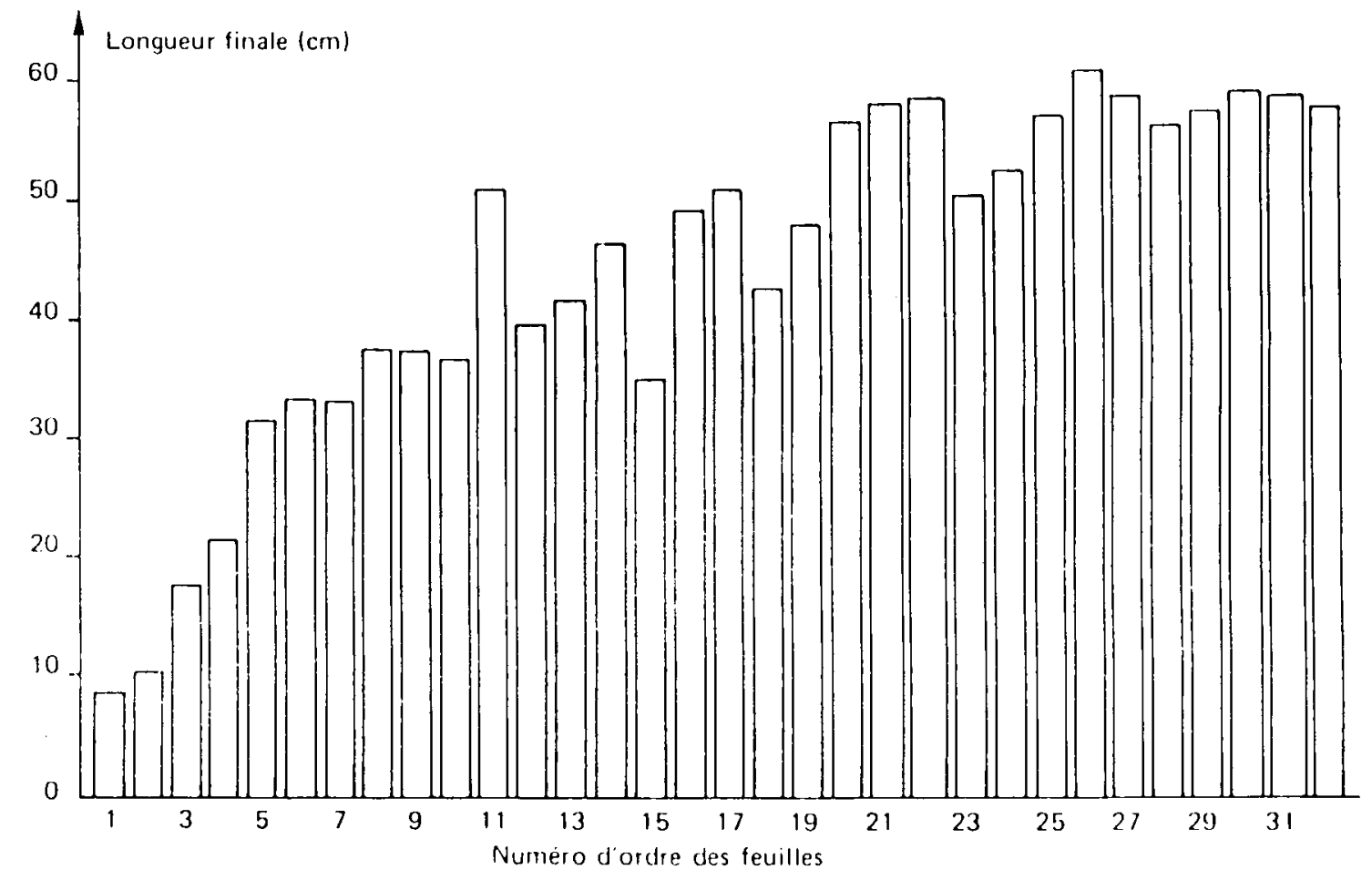

Fig. 1. Longueur finale des feuilles en fonction de leur numéro d'ordre chez les plantes à développement strictement végétatif. Plantes du cv Darmor (vernalisation préalable de 2 semaines). 


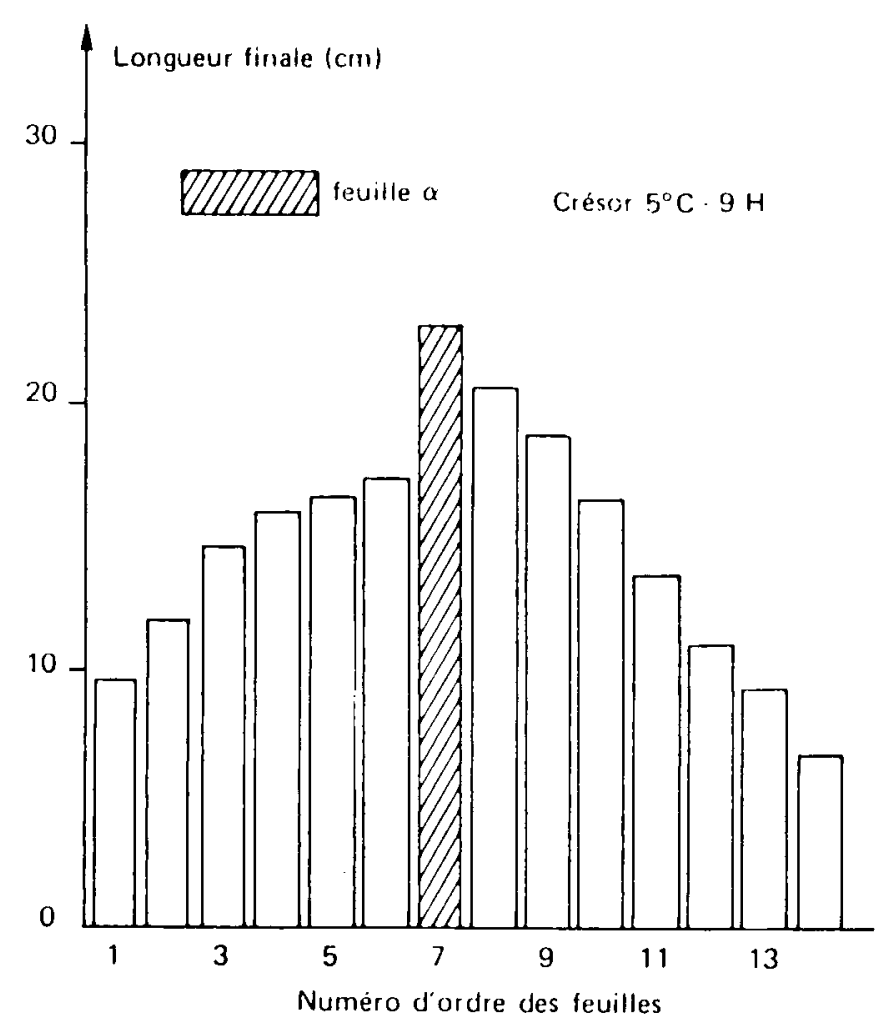

Fig. 2. Longueur finale des feuilles en fonction de leur numéro d'ordre chez les plantes à développement inflorescentiel. (Plantes du cv Crésor maintenues à une température constante de $5^{\circ} \mathrm{C}$ en jours courts - vernalisation parallèle.)

gement augmente jusqu'à une valeur maximale atteinte pour la feuille précédant la feuille $\alpha$, puis diminue progressivement pour les feuilles suivantes.

\section{Conclusion}

Ainsi, l'observation des trois caractères forme, longueur et vitesse d'allongement distingue les plantes végétatives des plantes inflorescentielles ayant subi la vernalisation préalable. Les feuilles de ces dernières se divisent, quant à elles, en deux séries avec la feuille $\alpha$ comme feuille charnière.

La comparaison avec les dissections montre que ces feuilles $\alpha$ sont initiées au moment de la déformation de l'apex en dôme. Lorsque les conditions sont favorables, ce passage se fait rapidement et il n'y a qu'une feuille au limbe acuminé et les feuilles suivantes se simplifient rapidement.

En revanche, si les conditions sont moins favorables, le passage peut s'étaler sur 15 jours et il $y$ a plusieurs feuilles au limbe légèrement acuminé. Bien que ces feuilles soient très semblables, seule la première d'entre elles est appelée feuille $\alpha$. Le passage vers la bractée s'étale sur plusieurs feuilles successives.

\section{Vitesses d'émission des feuilles}

Elles sont mesurées par l'intermédiaire des calculs des plastochrones réels et apparents qui permettent de connaître le temps séparant respectivement l'initiation et l'apparition des primordia foliaires. Ce plastochrone nous est donné par la pente de la droite de régression.

\section{Plastochrone réel}

Chez les plantes à développement strictement végétatif (Fig. 4A), les points s'ajustent bien à une droite $(r>0.90)$. Ainsi, la durée nécessaire à l'initiation de 2 primordia foliaires successifs est constante au cours de la croissance. Par exemple, pour les plantes du cultivar Darmorvernalisées 2 semaines, cette durée est de 3 jours. Les résultats sont similaires pour toutes les autres expériences menées sur des plantes à développement strictement végétatif.

Par contre, chez les plantes devenues inflorescentielles, les points représentant l'évolution du nombre total moyen de feuilles ne s'ajustent plus à une seule droite. II existe une phase d'accélération induisant un point de rupture; nous schématiserons la relation par une sigmoïde.

Par exemple, pour les plantes du cv Darmor 

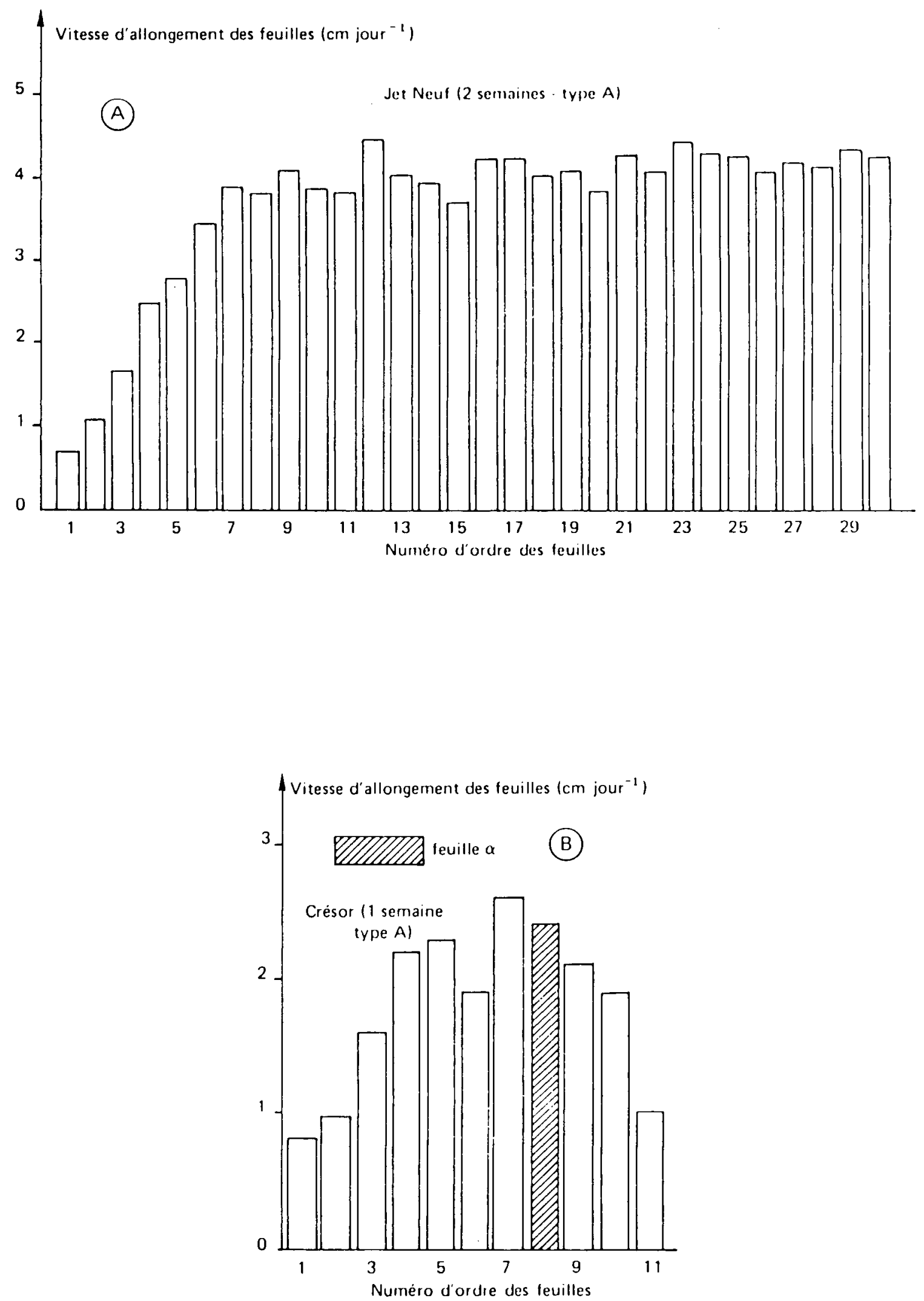

Fig. 3. Vitesse d'allongement des feuilles en fonction de leur numéro d'ordre. A. Plantes à développement strictement végétatif - Jet Neuf 2 semaines de vernalisation préalable. B. Plantes à développement inflorescentiel - Crésor 1 semaine de vernalisation préalable. 

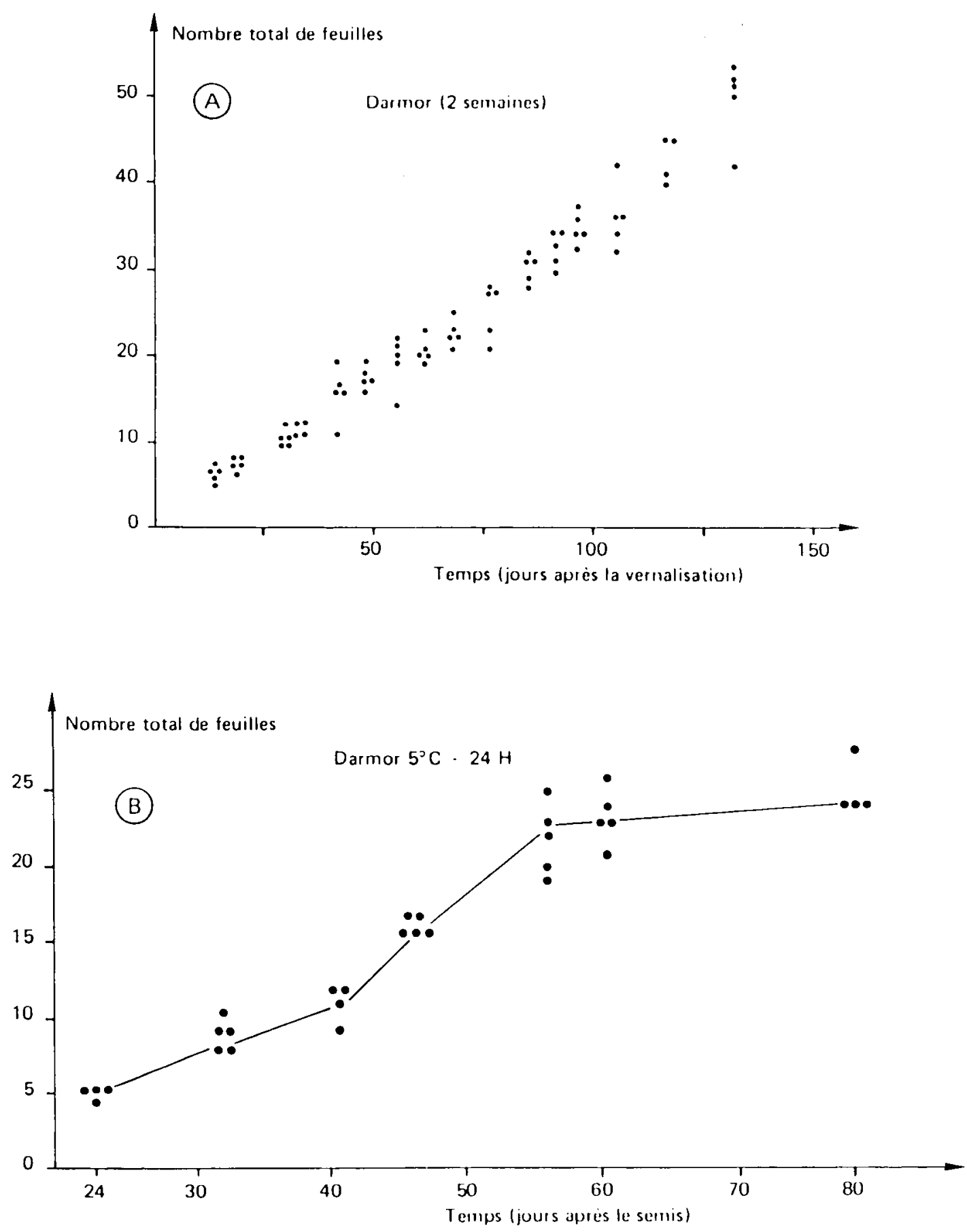

Fig. 4. Evolution du nombre total de feuilles. A. Plaíies à développement végétatif. Darmor 2 semaines de vernalisation préalable. B. Plantes à développement inflorescentiel - Darmor $5^{\circ} \mathrm{C}$ vernalisation parallèle.

(Fig. 4B) cultivées à une température constante de $5^{\circ} \mathrm{C}$ et en jours continus, l'intervalle de temps séparant l'initiation de 2 primordia foliaires successifs est dans un premier temps de 3,7 jours puis ce plastochrone réel moyen diminue et passe à une valeur de 1,4 jour. Cette deuxième valeur est constante jusqu'à son annulation au moment de l'initiation florale.

Les dissections réalisées au cours du temps, ont permis de préciser que cette augmentation rapide du nombre total de feuilles survient au moment du gonflement de l'apex en dôme (Tableau I).

\section{Plastochrone apparent}

L'accroissement de l'âge foliaire est linéaire au cours du temps chez les plantes à développement strictement végétatif (Fig. 5A). Par contre, chez les plantes devenues inflorescentielles, le rythme d'émergence des feuilles s'ac- 
célère à un moment donné (Fig. 5B). Chez le cv Jet Neuf, vernalisé 8 semaines, cette accélération se réalise lors de l'émergence de la feuille d'ordre 5. Les observations effectuées sur ces plantes indiquent que cette feuille d'ordre 5 correspond à la feuille $\alpha$. Les résultats sont similaires pour toutes les plantes exposées dans des conditions favorables au développement inflorescentiel (Tableau I).

L'émergence de cette feuille $\alpha$ est donc liée à une diminution du phyllochrone.

\section{Cas des ramifications florifères}

Cette feuille $\alpha$, avec toutes ses caractéristiques, a une position déterminée. En effet, sa présence est liée au devenir inflorescentiel et à l'architecture de la plante en pleine floraison. Dans nos conditions expérimentales, les ramifications latérales florifères ne se développent pas à l'aisselle de toutes les feuilles mais selon un gradient strictement basipète et essentiellement à l'aisselle des feuilles lancéolées. Parfois elles peuvent descendre jusqu'à la feuille $\alpha$, jamais en dessous.

\section{Discussion et Conclusion}

A partir du stade cotylédonnaire, les plantes n'ont subi qu'un seul climat. De ce fait nous pensons que les évolutions constatées sont bien liées à la floraison et non à des artéfacts climatiques.

Ainsi, la mise à fleurs des plantes de $B$. napus est liée à une évolution foliaire caractérisée par 2 phases successives : Phase 1:1) aug- mentation de la complexité morphologique et de la longueur finale des feuilles; 2) augmentation de leur vitesse d'allongement; 3) absence de ramifications latérales secondaires. Cette phase est semblable à celle des plantes à développement strictement végétatif. Phase II : 1) simplification de la forme et diminution de la longueur finale des feuilles; 2) diminution de leur vitesse d'allongement; 3) diminution des plastochrones réels et apparents; 4) présence de ramifications latérales secondaires.

Le passage de la phase I à la phase II peut être caractérisé par une feuille de transition, appelée $\alpha$ et dont les principales caractéristiques sont les suivantes : 1) son initiation s'effectue lors du gonflement de l'apex qui s'accompagne d'une accélération de l'initiation foliaire (réduction du plastochrone réel); 2) son émergence est liée à une accélération de l'émergence des feuilles suivantes (réduction du plastochrone apparent); 3) sa longueur finale est maximale; 4) elle marque le début du ralentissement de la vitesse d'allongement des feuilles.

Toutes les modifications observées chez les 3 cvs de $B$. napus et liées à la floraison représentent un phénomène général observé. En 1981, Bernier et al. ont regroupé l'ensemble de ces modifications sous la dénomination de "syndrome floral"s.

Nos observations sur le colza ne nous ont permis de retrouver que trois de ces modifications : 1) élargissement et déformation en dôme du méristème apical; 2) augmentation du taux d'initiation foliaire (diminution du plastochrone réel). Ceci est un phénomène assez courant que l'on

Tableau I. Pour quelques expériences prises au hasard, relation entre le numéro d'ordre de la feuille $\alpha$ et le numéro d'ordre de la feuille qui apparaît au moment de l'accélération de l'émergence de feuilles.

\begin{tabular}{|c|c|c|c|c|c|c|}
\hline \multicolumn{2}{|c|}{ Expérimentation } & \multirow{2}{*}{$\begin{array}{l}\text { Numéro } \\
\text { d'ordre de } \\
\text { la feuille } \alpha\end{array}$} & \multirow{2}{*}{$\begin{array}{l}\text { Numéro d'ordre } \\
\text { de la feuille } \\
\text { initiée au moment } \\
\text { du gonflement } \\
\text { de l'apex } \\
8\end{array}$} & \multirow{2}{*}{$\begin{array}{l}\text { Numéro d'ordre de } \\
\text { la feuille qui apparaît } \\
\text { au moment de } \\
\text { l'accélération } \\
\text { de l'émergence } \\
8\end{array}$} & \multicolumn{2}{|c|}{$\begin{array}{l}\text { Valeur des } \\
\text { plastochrones } \\
\text { (en jours) }\end{array}$} \\
\hline Cresor & $\begin{array}{l}5^{\circ} \mathrm{C}-9 \mathrm{~h} \\
2 \mathrm{sem} . \mathrm{de}\end{array}$ & & & & 5,1 et 2 & - \\
\hline & vernal. de type B & 6 & 6 & 6 & - & 一 \\
\hline Jet Neuf & $\begin{array}{l}4 \text { sem. de } \\
\text { vernal. de type } A\end{array}$ & 9 & 9 & 9 & 7,5 et 1,34 & 2,66 et 1,37 \\
\hline Darmor & $\begin{array}{l}5^{\circ} \mathrm{C}-24 \mathrm{~h} \\
8 \mathrm{sem} . \mathrm{de}\end{array}$ & 11 & 12 & 12 & 3,7 et 1,4 & - \\
\hline & vernal. de type B & 16 & 16 & 16 & 一 & 一 \\
\hline
\end{tabular}



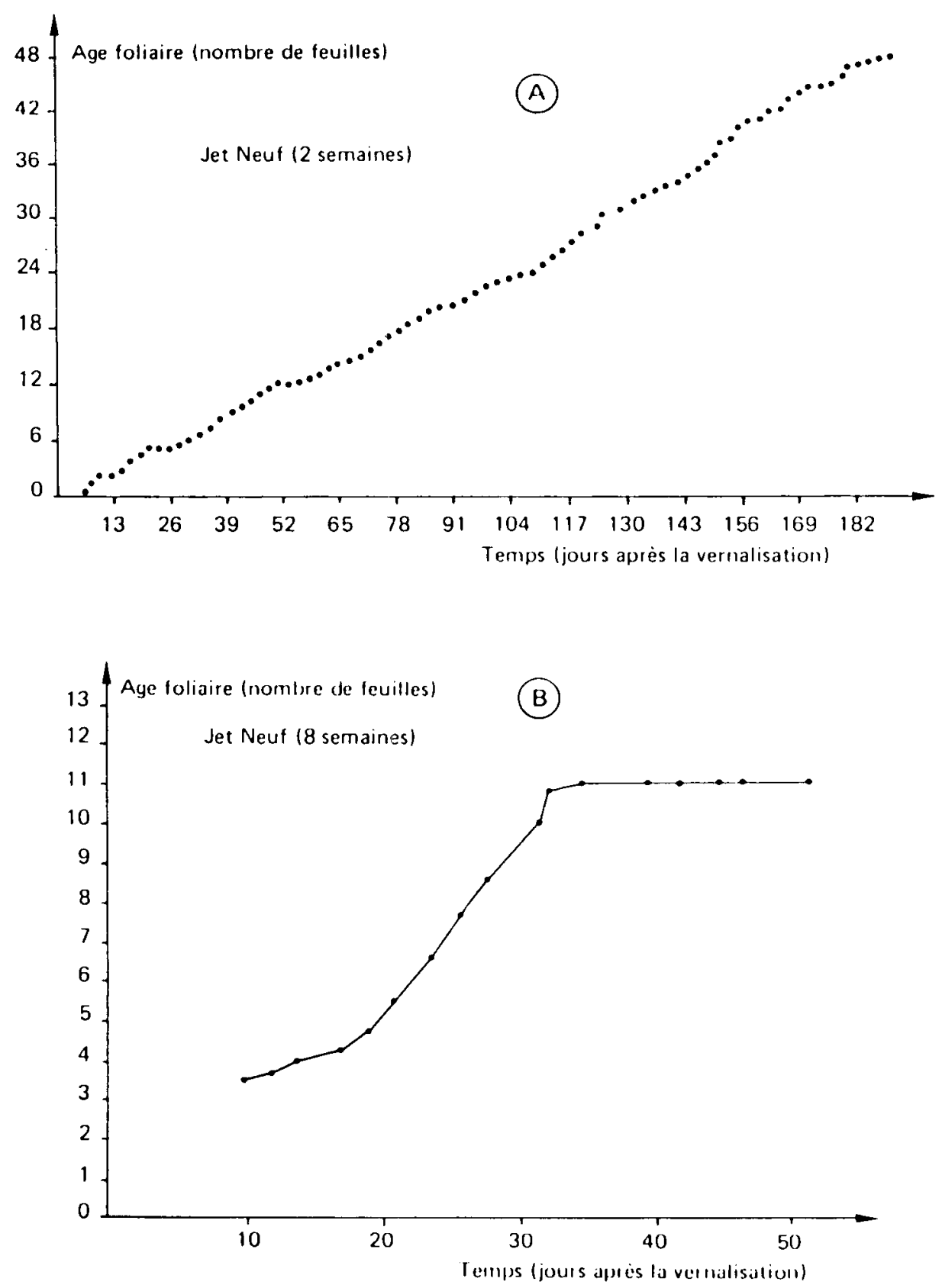

Fig. 5. Evolution de l'âge foliaire en fonction du temps. A. Plantes végétatives. Jet Neuf 2 semaines de vernalisation préalable. B. Plantes inflorescentielles. Jet Neuf 8 semaines de vernalisation préalable.

retrouve pendant la transition florale aussi bien pour les plantes de jour long que de jour court (Lager et Bussel, 1964; Vincent, communication personnelle). Letoublon (1985) et Chaput (1986) ont cependant du mal à retrouver ce phénomène chez le colza en plein champ; 3) changement de forme des feuilles.

Les modifications des caractéristiques foliaires observées lors de la phase de transition dépendraient de l'état structural du méristème au moment de l'initiation de ces feuilles. Ces résultats aboutissent à la même conclusion que Gif- ford et Tepper (1961) sur Chenopodium album ou Bernier (1964) sur Sinapis alba. Ces dimensions pourraient être dues à une compétition visà-vis des assimilats entre les primordia foliaires initiés.

Les primordia foliaires sont dépendants des assimilats produits par les feuilles émergées. Au sein du bourgeon terminal, ces primordia sont plus nombreux du fait de la diminution du plastochrone réel. La multiplication ou l'allongement cellulaire des primordia foliaires serait alors ralenti. II en découlerait une vitesse d'allonge- 
ment de la feuille plus faible et un nombre de cellules moindre donc une longueur finale de la feuille plus petite.

II nous paraît important de souligner la synchronisation de tous les événements observés. Cette synchronisation peut s'expliquer simplement à partir des travaux de Bernier (1964) et de Sachs (1969), selon lesquels l'état d'un méristème arrête le devenir de la feuille en cours d'initiation. Pour ces deux auteurs, ce devenir ne concernait que la forme; nous montrons qu'il peut s'étendre de sa vitesse d'allongement, à sa longueur finale, à son plastochrone et à sa possibilité d'axiller un rameau florifère. Cette synchronisation est précieuse car elle permet simplement par l'observation des feuilles, de remonter à l'état du méristème de départ.

Les résultats obtenus par Netzer (1986) concernant le nombre final de feuilles de l'axe principal chez $B$. napus mettent en évidence qu'il existe une relation entre ce nombre final de feuilles et le numéro d'ordre de la feuille $\alpha$ et par là même le nombre potentiel de ramifications latérales.

Cette corrélation peut être modélisée sous la forme $N_{\mathrm{t}}=K \alpha$; avec $N_{\mathrm{t}}=$ nombre final de feuilles sur l'axe principal; $K=$ constante; $\alpha=$ numéro d'ordre de la feuille $\alpha$.

Dans nos conditions expérimentales décrites précédemment, et sur l'ensemble des expérimentations, $K=1,84 \pm 0,33(1,51<K<2,17)$ quel que soit le cultivar considéré.

Au champ, Letoublon (1985) met en évidence la présence d'une feuille de transition morphologiquement différente de la feuille $\alpha$ précédemment décrite dans les conditions du phytotron. Cette feuille pourrait toutefois jouer un rôle analogue et la valeur du coefficient $K$ est de 1,96 \pm 0,22 soit $1,74<K<2,18$.

Ainsi, il nous est possible de conclure que, connaissant le numéro d'ordre de la feuille $\alpha$ (qui apparaît et se reconnaît très précocement), nous pouvons en déduire le nombre final de feuilles de l'axe principal et le nombre potentiel des ramifications puisque ces dernières ne se développent qu'à partir de la feuille $\alpha$.

Ce nombre final de feuilles est une composante importante de l'indice foliaire, qui lui même est un élément descriptif de la variabilité des rendements entre lieux et entre années.

\section{Remerciements}

Les auteurs remercient le CETIOM qui a financé cette étude et le phytotron de Gif-sur-Yvette qui leur a permis de la réaliser.

\section{Références}

Bernier G. (1964) Etude histophysiologique et histochimique de l'évolution du méristène apical de Sinapis alba L. cultivé en milieu conditionné et en diverses durées de jours favorables ou défavorables à la mise à fleurs. Mem. Acad. Roy. Belg. cl. Sci. XVI, I-XI et 1.150

Bernier G., Kinet J.M. \& Sach R.M. (1981) The physiology of flowering II. Transition to reproductive growth in C.R.C. press

Chaput J.P. (1986) Stades de développement chez le colza d'hiver Brassica napus L. oleifera Metzg. Phase préflorale - Montaison - Floraison. Mémoire de fin d'étude, ENITA de Dijon

Gifford E.M. \& Tepper M.B. (1961) Ontogeny of the inflorescence in Chenopodium album. Can. J. Bot. 48, 657-667

Lager R.H.M. \& Bussel W.T. (1964) The effect of flower induction on the rate of leaf initiation. Ann. Bot. 28, 163

Letoublon F. (1985) Evénements liés à la floraison chez le colza d'hiver (Brassica napus L.). Mém. fin d'étude, ENITA de Dijon

Malvoisin P. (1984) Organogenèse et croissance du maître-brin du blé tendre (Triticum aestivum) du semis à la floraison. I. Relations observées entre la croissance foliaire et la différenciation des ébauches foliaires ou florales. Agronomie 4, 557-564

Netzer M.H. (1986) Recherches sur le déterminisme de la floraison de 3 cvs de Brassica napus $L$. oleifera en conditions contrôlées. Thèse $3^{e}$ cycle, Univ. P. et M. Curie

Netzer M.H., Tittonel E.D. \& Merrien A. (1987) Initiation florale du colza d'hiver Brassica Napus L. cv Jet Neuf en conditions contrôlées. Inf. Tech. CETIOM 96, p. 39

Sachs T. (1969) Regeneration experiments on the determination of the forms of the leaves. Isr. J. Bot. 18, 21-30

Tittonel E., Desplantes G., Grangeret I. \& Pinochet X. (1982) Modifications morphologiques d'un bourgeon de colza (Brassica napus) au cours de la formation des ébauches florales. Inf. Tech. CETIOM 78, 15-24 